\title{
Revacept: Collagen GP VI receptor inhibitor, a new \& a formidable target for antiplatelet action
}

\author{
Sangeeta Bhanwra ${ }^{1}$, Kaza Ahluwalia ${ }^{1}$, Kavita Sekhri ${ }^{2}$
}

\begin{abstract}
${ }^{1}$ Department of Pharmacology, Government Medical College \& Hospital, Sector- 32, Chandigarh, India ${ }^{2}$ Department of Pharmacology, Dr. Harvansh Singh Judge Institute of Dental Sciences, Panjab University, Chandigarh, India
\end{abstract}

Received: 10 February 2013

Accepted: 15 March 2013

\section{*Correspondence to: \\ Dr. Sangeeta Bhanwra, \\ Email: doc_sangeeta@yahoo.com}

(C) 2013 Bhanwra S et al. This is an open-access article distributed under the terms of the Creative Commons Attribution License, which permits unrestricted use, distribution, and reproduction in any medium, provided the original work is properly cited.

\section{INTRODUCTION}

Platelets play an important role in the pathophysiology of coronary heart disease. In the atherosclerotic vessels, platelet activation, adhesion and aggregation occurs after plaque rupture or vessel wall injury leading to the thrombotic and ischemic complications in coronary heart disease. ${ }^{1}$ At sites of vascular injury, collagen-mediated platelet adhesion and activation is one of the first events in platelet-dependent thrombus formation. Platelet recruitment at sites of vascular injury is crucial for normal haemostasis and efficient wound healing. At these sites, exposed collagen is one of the most thrombogenic substrates. Interaction of platelets with collagen leads to platelet activation, resulting in platelet shape change, generation of thromboxane A2 and secretion of granular content e.g. ADP, and activation of integrin aIIb $\beta 3$. These events result in the recruitment of additional platelets to the growing platelet plug and finally platelet aggregation. Because of its prominent role in thrombogenesis, the platelet-collagen axis holds great promise as a therapeutic target to prevent platelet-dependent thrombus formation. However, there are different types of collagen present in the vessel wall, and several collagen receptors too, making collagen-dependent thrombus formation an intricate interplay between different components. ${ }^{2}$

\begin{abstract}
Antiplatelet drugs play an important role in the prevention as well as treatment of cardiovascular diseases like coronary artery disease and stroke. Many of the currently available antiplatelet drugs face limitations due to safety and efficacy issues. A new antiplatelet drug, revacept i.e. a collagen receptor antagonist, has been shown to reduce platelet adhesion by blocking GP VI-dependent pathways without increasing the risk of bleeding complications and without affecting the general hemostasis.
\end{abstract}

Keywords: Platelet adhesion, Revacept, Collagen GP VI receptor inhibitor

In recent years, a number of high-performing antiplatelet drugs have been developed, but most of these agents had the accompanying disadvantage of increased bleeding risk, even though they proved themselves effective in reducing the ischemic events. These findings indicate a need for a compound with the potential to inhibit platelet adhesion and activation and collagen aggregation without affecting general hemostasis. It was seen that the compounds that specifically interfere in the collagen induced platelet activation, thereby inhibiting platelet aggregation, reduce the risk of complications considerably. ${ }^{1}$

\section{PLATELET COLLAGEN INTERACTION}

The interactions between platelets and collagen supposedly play a significant role in the arteries or diseased vessels which have medium to high shear rates as there, platelets, will need a firm clinging base, on the exposed endothelium, to bind to and start the process of coagulation. ${ }^{3}$ There are three major collagen receptors responsible primarily for the platelet adhesion to the collagen and they are: GPVI, $\alpha 2 \beta 1$ and GP Ib-V-1X. ${ }^{4}$ It is now very well recognized that GPVI receptors are the main receptors which provide the firm base for platelet adhesion under the high shear stress conditions, which is 
further reinforced by the release of soluble mediators like adenosine diphosphate (ADP) and thromboxane A2 (TxA2), and thrombin. This new appreciation of the role of GPVI in mediating integrin activation has now made GPVI the critical role player in the initial collagenplatelet interaction, upstream of adhesion. ${ }^{3}$ It can be inhibited either by using the antibodies directed against it e.g. JAQ1, or by using a soluble form of the receptor, which acts as a competitive inhibitor, e.g. revacept. ${ }^{2}$ However the problem with JAQ1 was that it caused the down regulation of GPVI and also reduced the platelet count, thereby leading to bleeding risk. This was not seen with revacept. ${ }^{5}$ Hence revacept, an inhibitor of GPVI holds promise as an antiplatelet agent that potently inhibits collagen induced aggregation of human platelets but not thrombin- or ADP-induced aggregation, thereby having minimal bleeding risk. ${ }^{1}$

\section{REVACEPT}

Revacept is a fusion protein consisting of an extracellular portion of GPVI receptor of humans for collagen, Fc portion of human IgG1, a small linker protein sequence and a hinge type portion from human IgG1. ${ }^{1}$ Hence, revacept (PR-15) is technically a dimeric glycoprotein [GP] VI-Fc and is almost equally effective as the other known antiplatelet drugs, without any additional complication of bleeding as seen in various animal studies. ${ }^{5}$ Revacept acts against collagen which gets exposed in endothelial lesions, and hence inhibits the activation of the platelet receptors which bind to collagen and start the platelet aggregation process. Therefore, revacept acts at a very early stage in acute vascular syndromes. Revacept potently inhibits collagen induced aggregation of human platelets but not thrombin- or ADP-induced aggregation but does neither induce down regulation of the platelet receptor nor reduced platelet count. ${ }^{1}$

\section{CLINICAL TRIALS}

The phase I clinical trial done on revacept showed it to be safe and tolerable. The drug showed linear pharmacokinetic profile and a very specific pharmacodynamic profile. There was no effect either on general hemostasis or on coagulation, as reflected by monitoring bleeding times, activated partial thromboplastin time and international normalized ratio. Also, inspite of being a protein derived drug, it didn't generate any anti-revacept antibodies even after 6 weeks of its administration. Revacept initially decreased rapidly from the blood, leading to low serum levels followed by a gradual, much slower decrease in concentration after that. ${ }^{1}$ Various studies done on animals had proved the superiority of revacept, as regards to the effect and safety profile, when compared to the other anti GPVI antibodies or the Fc GPVI proteins. ${ }^{1,5-7}$ The drug is currently under phase 2 trials in humans. ${ }^{8}$

\section{PHARMACOKINETICS AND PHARMACODYNAMICS}

The pharmacokinetic of revacept is linear, with concentration and AUC increasing with the increase in the dose. The elimination is slow, with terminal half life ranging from 60-130 hours. Revacept is primarily distributed in the systemic circulation with volume of distribution at steady state ranging between 7.8-12.3 L. The route of administration is intravenous.

After the administration of revacept, there occurs a strong and clearly significant time- and dose-dependent inhibition of collagen-induced aggregation. The effect starts within 2 hours of starting the infusion, and lasts for as long as 24 hours after stopping the infusion. This prolonged effect was seen because of the inhibition of collagen mediated platelet activation and aggregation, without having any effect on ADP- or thrombin induced aggregation. It takes a lot of time, almost few weeks, for the platelet activity to return to the normal levels, after giving revacept. ${ }^{1}$

\section{SAFETY PROFILE OF REVACEPT}

No significant effect is seen on general coagulation and bleeding times, indicating that Revacept is relatively better, when it comes to safety, than the current antiplatelet drugs. The phase 1 study of revacept in humans didn't show any serious adverse effects related to it, except tonsillitis, nasopharyngitis etc, which were seen in few subjects. No anti-revacept antibodies were seen, even up to six weeks of administration. ${ }^{1}$

\section{PLACE IN THERAPY}

Inspite of all the understanding about the mechanisms of plaque-mediated arterial thrombosis, current antithrombotic concepts rely solely on the prevention of platelet aggregation by fibrinogen receptor antagonists, aspirin, and P2Y12 antagonists. The consequences of platelet activation and aggregation are targeted, and not the source, with the resultant drugs having lesser efficacy and safety. Recent clinical data with intravenous GPIIbIIIa inhibitors e.g. abciximab etc. and newer antiplatelet agents e.g. prasugrel showed that these drugs, though efficacious as antiplatelet agents, were increasingly associated with higher bleeding complications. Since, GPVI plays an important role in initial platelet adhesion and thereby initiating the signalling cascade which ultimately causes platelet aggregation and thrombus formation, a drug which can inhibits this protein will have strong antithrombotic effect. Revacept is basically a dimeric protein formed from the fusion of soluble portion of the GPVI receptor and human $\operatorname{IgG1}$, which scavenges the collagen and inhibits fibronectin at the site of atherosclerotic plaque, thereby acting at a very early stage in the pathogenesis of acute vascular syndromes. Moreover it doesn't cause increased bleeding risk unlike the currently available 
antiplatelet drugs. It is basically the result of the concept of lesion directed inhibition of thrombus formations and revacept seems to hold a lot of promise for development into a safe and highly efficacious antiplatelet drug for the treatment of thrombotic disorders. Apart from the antiplatelet effect, revacept seems to have an additional anti- atherosclerotic effect by inducing the remodelling of vessel endothelium in arteries with prior catheterization on long term use. ${ }^{9}$ Thus, revacept holds a promise to having long term benefits in atherosclerosis patients, along with the short term benefit as an antithrombotic agent. This will play an important role in warding off various athero-thrombotic complications on which, the currently available antithrombotic agents have either none or a little beneficial effect. ${ }^{1}$

\section{Funding: No funding sources}

Conflict of interest: None declared

Ethical approval: Not required

\section{REFERENCES}

1. Ungerer M, Rosport K, Bültmann A, Piechatzek R, Uhland K, Schlieper P, et al. Novel antiplatelet drug revacept (Dimeric Glycoprotein VI-Fc) specifically and efficiently inhibited collagen-induced platelet aggregation without affecting general hemostasis in humans. Circulation 2011;123:1891-9.

2. Deckmyn H, De Meyer SF, Bross K, Vanhoorelbeke $\mathrm{K}$. Inhibitors of the interactions between collagen and its receptors on platelets. Handb Exp Pharmacol 2012;(210):311-37.
3. Nieswandt B, Watson SP. Platelet-collagen interaction: is GPVI the central receptor? Blood 2003;102:449-61.

4. Xiang YZ, Xia Y, Gao XM, Shang HC, Kang LY, Zhang BL. Platelet activation, and antiplatelet targets and agents: current and novel strategies. Drugs 2008;68:1647-64.

5. Gruner S, Prostredna M, Koch M, Miura Y, Schulte V, Jung SM, et al. Relative antithrombotic effect of soluble GPVI dimer compared with anti-GPVI antibodies in mice. Blood 2005;105:1492-9.

6. Gruner S, Prostredna M, Aktas B, Moers A, Schulte V, Krieg T, et al. Anti-glycoprotein VI treatment severely compromises hemostasis in mice with reduced alpha2beta1 levels or concomitant aspirin therapy. Circulation 2004;110:2946-51.

7. Schulte V, Reusch HP, Pozgajova M, Varga-Szabo D, Gachet C, Nieswandt B. Two-phase antithrombotic protection after anti-glycoprotein VI treatment in mice. Arterioscler Thromb Vasc Biol 2006;26:1640-7.

8. Poppert H. Revacept in Symptomatic Carotid Stenosis (Revacept/CS/02). Available at http://clinicaltrials.gov/show/NCT01645306. NLM Identifier: NCT01645306. Accessed 8 February 2013.

9. Bultmann A, Li Z, Wagner S, Peluso M, Schonberger $\mathrm{T}$, Weis $\mathrm{C}$, et al. Impact of glycoprotein VI and platelet adhesion on atherosclerosis: a possible role of fibronectin. J Mol Cell Cardiol 2010;49:532-42.

doi:10.5455/2319-2003.ijbcp20130624

Cite this article as: Bhanwra S, Ahluwalia K, Sekhri K. Revacept: Collagen GP VI receptor inhibitor, a new \& a formidable target for antiplatelet action. Int $\mathbf{J}$ Basic Clin Pharmacol 2013;2:344-6. 